ISSN 1997-5902

\title{
Valorisation des plantes médicinales à coumarines des marchés de Douala Est (Cameroun)
}

\author{
MPONDO MPONDO Emmanuel ${ }^{1}$, YINYANG Jacques*1 ${ }^{*}$, DIBONG Siegfried Didier(1,2), \\ (1) Département des Sciences Pharmaceutiques, Faculté de Médecine et des Sciences \\ Pharmaceutiques, Université de Douala, B.P. 2701 Douala, Cameroun \\ (2) Département de Biologie des Organismes Végétaux, Faculté des Sciences, B.P. 24157 Douala, \\ Cameroun \\ Auteur de la correspondance : yinyangi@yahoo.fr
}

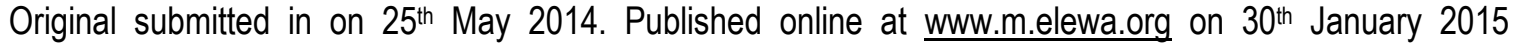
http://dx.doi.org/10.4314/jab.v85i1.6

\section{RESUME}

Objectif: Une enquête ethnobotanique a été conduite en février 2014 auprès des vendeuses des marchés Est de la ville de Douala concernant les différents usages des plantes à coumarines. Résultats : Au total, 38 espèces végétales à coumarines dont 25 herbacées et 13 ligneuses ont été inventoriées. Parmi ces espèces, 5 sont locales et 33 sont exotiques. Elles comprennent 34 genres repartis dans 21 familles dont la plus représentée est celle des Apiaceae. Le ravitaillement se fait dans les champs, les forêts secondarisées, les jardins de cases, vergers et jachères. Ces espèces sont pour la plupart cultivées et les feuilles, fruits et graines sont généralement utilisés pour la cuisine comme condiments ou comme épices. Parmi les maladies soignées par les plantes à coumarines recensées dans les marchés, les maladies de l'appareil digestif sont plus importantes $(33,33 \%)$. Les coumarines ont des propriétés antipyrétique, analgésique, sédative, antioedémateuse et anti convulsivante. Les plantes à coumarines et plus spécifiquement celles de la famille des Apiaceae ont la capacité de favoriser l'expulsion des gaz intestinaux entraînant une diminution des ballonnements et des flatulences. Elles augmentent la production stomacale d'acide contribuant à une bonne désinfection du bol alimentaire. Les coumarines ont la capacité de prévenir la peroxydation des lipides membranaires et de capter les radicaux hydroxyles, superoxydes et peroxyles.

Conclusion et application des résultats : Toutes ces propriétés peuvent justifier l'intérêt accordé à ces plantes dans la médecine traditionnelle et leurs utilisations à des fins thérapeutiques, curatives et préventives.

Mots clés : Ethnobotanique, coumarines, thérapeutiques, Apiaceae

\begin{abstract}
Objective: An ethnobotanical survey was conducted in February 2014 with the vendors of the Eastern markets of the city of Douala on the different uses of coumarin plants.

Results: A total of 38 plant species including 25 coumarin herbaceous and woody 13 were surveyed. Of these, 5 are local and 33 are exotic. They include 34 genera distributed in 21 families most represented is that of Apiaceae. Refueling is done in the fields, secondary forests, orchards and fallow. These species are mostly cultivated and leaves, fruits and seeds are generally used for cooking as a condiment or as spices. Among the diseases treated by coumarin plants identified in the markets, diseases of the digestive system are higher (33.33\%). Coumarins have antipyretic,
\end{abstract}


analgesic, sedative, and anti convulsant antioedmatous. Coumarins plants and specifically those of the family Apiaceae have the ability to promote the expulsion of intestinal gas resulting in a decrease bloating and flatulence. They increase the production of stomach acid contributing to a good disinfection of the bolus. Coumarins have the ability to prevent the peroxidation of membrane lipids and capture hydroxyl radicals, superoxides and peroxyls.

Conclusion and application of the results: All these properties can justify the interest given to these plants in traditional medicine and their uses for therapeutic, curative and preventive purposes.

Keywords: Ethnobotany, coumarins, therapeutic, Apiaceae

\section{INTRODUCTION}

Les plantes, éléments vitaux de la diversité biologique servent essentiellement au bien être humain (Adjanohoun et al., 1994, Adjanohoun, 2000). Après avoir longtemps combattu la médecine traditionnelle, médecins et organismes de santé portent davantage un intérêt aux valeurs et à l'efficacité des traitements par les plantes. De nombreuses études scientifiques ont été entreprises afin d'étudier l'aspect botanique et thérapeutique de ces dernières et d'intégrer leurs propriétés médicinales dans un système de santé moderne en exploitant leurs principes actifs (Biyiti et al., 2004). Environ $80 \%$ de la population mondiale et plus de $90 \%$ de la population des pays en voie de développement y recourent pour les soins de première nécessité (Cunningham, 1993 ; Pei, 2001; Olsen, 2005; Jiofack et al., 2010; Dibong et al., 2011). Les enquêtes ethnobotaniques s'avèrent être l'une des approches fiables pour la découverte de nouveaux médicaments (Koné, 2009). Toutefois, il existe un danger de voir se perdre le savoir lié aux plantes médicinales qui possède une réelle valeur culturelle et compromettre à terme le développement de nouveaux médicaments pharmaceutiques (Adomou et al., 2012). L'inexistence des infrastructures sanitaires, la constante augmentation des prix de produits et prestations de santé, l'inaccessibilité aux médicaments génériques très souvent toxiques constituent des problèmes majeurs pour les populations rurales et même

\section{MATERIEL ET METHODES}

Site d'étude : Douala (latitude, $03^{\circ} 40^{\prime}-04^{\circ} 11^{\prime} \mathrm{N}$; longitude, $09^{\circ} 16^{\prime}-09^{\circ} 52^{\prime} \mathrm{E}$; altitude, $13 \mathrm{~m}$ ) a un climat qui appartient au domaine équatorial d'un type particulier dit " camerounien " qui se urbaines. Pourtant, la médecine à base des plantes apparaît comme l'alternative la plus appropriée pour combler les carences en besoins sanitaires dont les populations aspirent. De même, la connaissance des principes actifs de ces plantes serait un atout indéniable en vue de la valorisation et de la meilleure utilisation de ces Produits Forestiers Non Ligneux (PFNLs). Cependant, le potentiel des plantes pour la production de nouveaux médicaments est largement inexploité (Hamburger et Hostettmann, 1991). La présente étude a pour but de pallier à ces lacunes. Les coumarines sont très largement distribuées dans le règne végétal. $\mathrm{Ce}$ sont des substances naturelles, organiques et aromatiques constituées de neuf atomes de carbone caractérisées par le noyau 2H-1benzopyrane-2-one (Gray et Waterman, 1978). Elles représentent avec les flavonoïdes, les chromones et les isocoumarines, un très vaste groupe de composés (Weterman et Grundon, 1983). Les coumarines simples dégagent une agréable odeur, rappelant la vanilline et ont été utilisées pour la conception des parfums et arômes de synthèse. L'objectif principal de cette étude est de valoriser la pharmacopée traditionnelle des plantes à coumarines vendues dans les marchés Est de la ville de Douala. Les objectifs spécifiques envisagés sont : (1) de recenser les plantes à coumarines connues par les populations de la ville de Douala et (2) d'inventorier les différents usages.

caractérise par deux saisons avec une longue saison de pluies (au moins 9 mois), des précipitations abondantes (environ $4000 \mathrm{~mm}$ par an), des températures élevées $\left(26,7{ }^{\circ} \mathrm{C}\right)$ et 
stables. La moyenne minimale de température à Douala pour 30 années (1961-1990) est de 22,6 ${ }^{\circ} \mathrm{C}$ en juillet et la moyenne maximale de température de $32,3{ }^{\circ} \mathrm{C}$ en février. L'humidité relative de l'air reste élevée toute l'année et voisine de $100 \%$ (Din et al., 2008). Douala, chef-lieu de la région du Littoral compte quatre départements : le Moungo, la Sanaga Maritime, le Nkam et le Wouri. Douala a environ 2,5 millions d'habitants et la croissance démographique n'est pas toujours suivie d'une augmentation des ressources économiques (Priso et al., 2011). La région du Littoral est un ensemble d'écosystèmes où se côtoient les forêts denses humides sempervirentes notamment la forêt dense humide sempervirente littorale entre 0 et $100 \mathrm{~m}$ d'altitude et la forêt dense humide biafréenne entre 100 et $500 \mathrm{~m}$ d'altitude, la mangrove, les marécages, la végétation ripicole, les agro écosystèmes et la végétation plus ou moins anthropisée (Letouzey, 1985). Ces écosystèmes terrestres et hydromorphes subissent d'énormes pressions anthropiques (pollution surtout en milieu urbain et déforestation en milieu périurbain) (Priso et al., 2011).

Enquêtes ethnobotaniques: La liste des plantes à coumarines a été dressée à partir de la documentation botanique et ethnopharmacologique disponibles. Cette recherche a été confirmée sur le terrain par la localisation de chaque espèce dans son site écologique. Des enquêtes ethnobotaniques basées sur les interrogations directes portant sur les usages des plantes citées dans la pharmacopée traditionnelle ont été conduites durant le mois de février 2014 auprès des vendeuses des marchés Est de la ville de Douala. L'approche des vendeuses dont l'âge varie entre 30 et 45 ans a été basée sur le dialogue en langues française et locale. 40 vendeuses ont été enquêtées soient 10 par marché. Ces dernières devaient donner toutes les informations relatives aux plantes à coumarines. Pour chaque vendeuse qui acceptait répondre aux questions et pour une maladie donnée, les informations ont été collectées selon un canevas standardisé inspiré des fiches Pharmel (Adjanohoun et al., 1989,

\section{RESULTATS ET DISCUSSION}

Au total, 38 espèces végétales à coumarines dont 25 herbacées et 13 ligneuses ont été inventoriées auprès des vendeuses des marchés Est de la ville de Douala. Les herbacées sont : Afromomum escarpum (Sims) Hepper, Allium cepa Linn., Albelmoschus esculentus (Linn.) Moench, Allium
1994). Pour chaque indication thérapeutique, les détails de la recette sont relevés avec soins et la plante concernée est présentée.

Les descriptions botaniques et les différents usages des plantes médicinales rencontrées ont été complétés par des informations fournies par des auteurs tels que Letouzey (1970), Béllé (1992). Les échantillons ont été récoltés et conservés selon les techniques et méthodes de Dibong et al.(2011). Les étudiants du niveau I du Département de Sciences Pharmaceutiques de la Faculté de Médecine et des Sciences Pharmaceutiques de l'Université de Douala ont été mis à contribution pour cette étude. Ils ont été répartis en 04 groupes de 20 étudiants soient 10 personnes enquêtées par groupe. Dans chaque groupe, la présence d'au moins un étudiant appartenant à la tribu majoritaire du marché choisi a permis de communiquer et de réaliser sans difficulté majeure les enquêtes auprès des vendeuses sollicitées. Les informations sur le " diagnostic des maladies " ont été complétées par la revue bibliographique (Adjanohoun et al., 1989; Adjanohoun, 2000). Pour une exploitation pratique des données et une harmonisation avec le système international, les problèmes de santé cités ont été distingués en grands groupes de maladies selon la classification des maladies proposée par l'Organisation Mondiale de la Santé (OMS, 2000) et adaptée par l'Organisation de l'Unité Africaine (OUA) pour la pharmacopée camerounaise (Adjanohoun et al., 1994) et suivant la classification statistique internationale des maladies et des problèmes de santé connexes version 2010 (WHO, 2010). Des fiches d'enquête ont été élaborées dans le but de recueillir notamment des informations sur les parties de la plante utilisées, les modes de préparation, les maladies soignées, le mode d'administration et la posologie.

Collecte des données: Les échantillons des plantes ont été récoltés et leur identification validée à l'herbier du jardin botanique de Limbé. Toutes les espèces nommées ont été répertoriées et identifiées. Les données de terrain ont été enregistrées sur un tableur Excel puis analysées.

sativum Linn., Aloe Vera Linn. Anethum graveolens Linn., Apium graveolens Linn., Artemisia absinthium Linn., Brassica oleracea (Linn.) H. Bailey, Cnicus benedictus Linn., Coriandrum sativum Linn., Cucumis sativus Linn., Daucus carota Wild., Desmodium triflorum (Linn.) 
Mpondo et al.. J. Appl. Biosci. Valorisation des plantes médicinales à coumarines des marchés de Douala Est (Cameroun)

DC., Galium odoratum (Linn.) Scop., Glycine max Linn., Ocimum basilicum Linn., Oryza sativa Linn., Panax ginseng A.C. Meyer, Petroselinum sativum (Mill.) Nymam ex A.W. Hill., Phaseolus vulgaris Linn., Pimpinella anisum Linn., Thymus vulgaris Linn., Urtica dioica Linn., Zea mays Linn. Les ligneuses sont : Carica papaya Linn., Citrus aurantifolia (Christm.) Swingle, Citrus grandis Osbeck, Citrus limon Linn., Citrus medica Linn., Combretum micrantum G.Don, Elaeis guineensis Jacq., Eurcalyptus sailgna Smith, Manihot esculenta Crantz, Persea americana Mill., Senna alata Linn., Sesamum indicum Linn., Vernonia amygdalina Del. 
Tableau 1 : Plantes à coumarines recensées dans les marchés Est de la ville de Douala.

\begin{tabular}{|c|c|c|c|c|c|c|}
\hline Familles & Noms scientifiques & $\begin{array}{l}\text { Noms } \\
\text { commerciaux }\end{array}$ & Maladies soignées & $\begin{array}{l}\text { Organes } \\
\text { utilisés }\end{array}$ & Mode de préparation & $\begin{array}{l}\text { Posologie et mode } \\
\text { d'administration }\end{array}$ \\
\hline \multirow[t]{12}{*}{ Apiaceae } & \multirow[t]{4}{*}{$\begin{array}{l}\text { Anethum } \\
\text { graveolens Linn. }\end{array}$} & \multirow[t]{4}{*}{ Fenouil } & $\begin{array}{l}\text { Manque d'appétit, } \\
\text { coliques, } \\
\text { spasmes intestinaux }\end{array}$ & $\begin{array}{l}\text { Feuilles, } \\
\text { fruits }\end{array}$ & $\begin{array}{l}\text { Infusion de } 50 \mathrm{~g} \text { de fruits et de } \\
\text { feuilles pendant } 60 \mathrm{mn}\end{array}$ & $\begin{array}{l}\text { Voie orale ( } 2 \text { tasses par } \\
\text { jour) }\end{array}$ \\
\hline & & & $\begin{array}{l}\text { Ballonnements et } \\
\text { maux d'estomac }\end{array}$ & Fruits & $\begin{array}{l}\text { Macérer les fruits frais dans } \\
\text { l'eau bouillante }\end{array}$ & Voie orale \\
\hline & & & Hypo lactation & Feuilles & Infusion & Voie orale \\
\hline & & & Diurèse difficile & Graines & $\begin{array}{l}\text { Infusion de la poudre de } \\
\text { graine (1-2 g, } 4 \text { à } 8 \mathrm{~g} \text { dans un } \\
\text { litre d'eau) }\end{array}$ & $\begin{array}{l}\text { Voie orale (boire } 2 \text { à } 3 \\
\text { verres par jour) }\end{array}$ \\
\hline & \multirow[t]{8}{*}{$\begin{array}{l}\text { Apium graveolens } \\
\text { Linn. }\end{array}$} & \multirow[t]{8}{*}{ Céleri } & $\begin{array}{l}\text { Hypertension } \\
\text { artérielle }\end{array}$ & Feuilles & $\begin{array}{l}\text { Décoction ( } 250 \mathrm{ml} \text { d'eau } \\
\text { chaude ou de } \\
\text { tisane d'huile essentielle }\end{array}$ & $\begin{array}{l}\text { Voie orale (boire } 2 \text { à } 3 \\
\text { verres par jour) }\end{array}$ \\
\hline & & & $\begin{array}{l}\text { Cycle menstruel } \\
\text { irrégulier }\end{array}$ & $\begin{array}{l}\text { Tige, } \\
\text { feuilles, } \\
\text { graines }\end{array}$ & $\begin{array}{l}\text { Pour déclencher le cycle, } \\
\text { écraser } 1,5 \text { cuillère à café } \\
\text { d'ache, verser } 1 \text { tasse d'eau } \\
\text { bouillante ; couvrir et laisser } \\
\text { macérer } 15-20 \mathrm{mn}\end{array}$ & $\begin{array}{l}\text { Voie orale (boire jusqu'à } \\
3 \text { tasses par jour) }\end{array}$ \\
\hline & & & $\begin{array}{l}\text { Intoxication } \\
\text { Alimentaire, } \\
\text { flatulence }\end{array}$ & Feuilles & $\begin{array}{l}\text { Décoction (1,5 I d'eau avec } \\
750 \mathrm{ml} \text { pendant } 30 \mathrm{mn} \text { ) }\end{array}$ & $\begin{array}{l}\text { Voie orale (boire } 1 \text { verre } \\
\text { tous les matins) }\end{array}$ \\
\hline & & & $\begin{array}{l}\text { Mal de nerf, } \\
\text { convulsion }\end{array}$ & Feuilles & $\begin{array}{l}\text { Décoction ( } 250 \mathrm{ml} \text { d'eau } \\
\text { chaude ou de tisane d'huile } \\
\text { essentielle) }\end{array}$ & $\begin{array}{l}\text { Voie orale (boire jusqu'à } \\
3 \text { tasses par jour) }\end{array}$ \\
\hline & & & Mauvais haleine & Feuilles & Crue ou en salade & Voie orale (mâcher) \\
\hline & & & Obésité & Graines & $\begin{array}{l}\text { Décoction ( } 250 \mathrm{ml} \text { d'eau } \\
\text { chaude ou de } \\
\text { tisane d'huile essentielle) }\end{array}$ & $\begin{array}{l}\text { Voie orale (boire jusqu'à } \\
3 \text { tasses par jour) }\end{array}$ \\
\hline & & & Asthme & Tige, feuilles & $\begin{array}{l}\text { Macérer et mélanger avec le } \\
\text { jus d'olive }\end{array}$ & $\begin{array}{l}\text { Voie orale pour adultes } \\
\text { (une cuillerée à soupe) ; } \\
\text { pour enfants, une } \\
\text { cuillerée à café }\end{array}$ \\
\hline & & & Goutte & Feuilles & $\begin{array}{l}\text { Infusion de } 60 \mathrm{~g} \text { de feuilles } \\
\text { pendant } 20 \mathrm{mn}\end{array}$ & $\begin{array}{l}\text { Voie orale ( } 3 \text { tasses par } \\
\text { jour loin des repas) }\end{array}$ \\
\hline
\end{tabular}




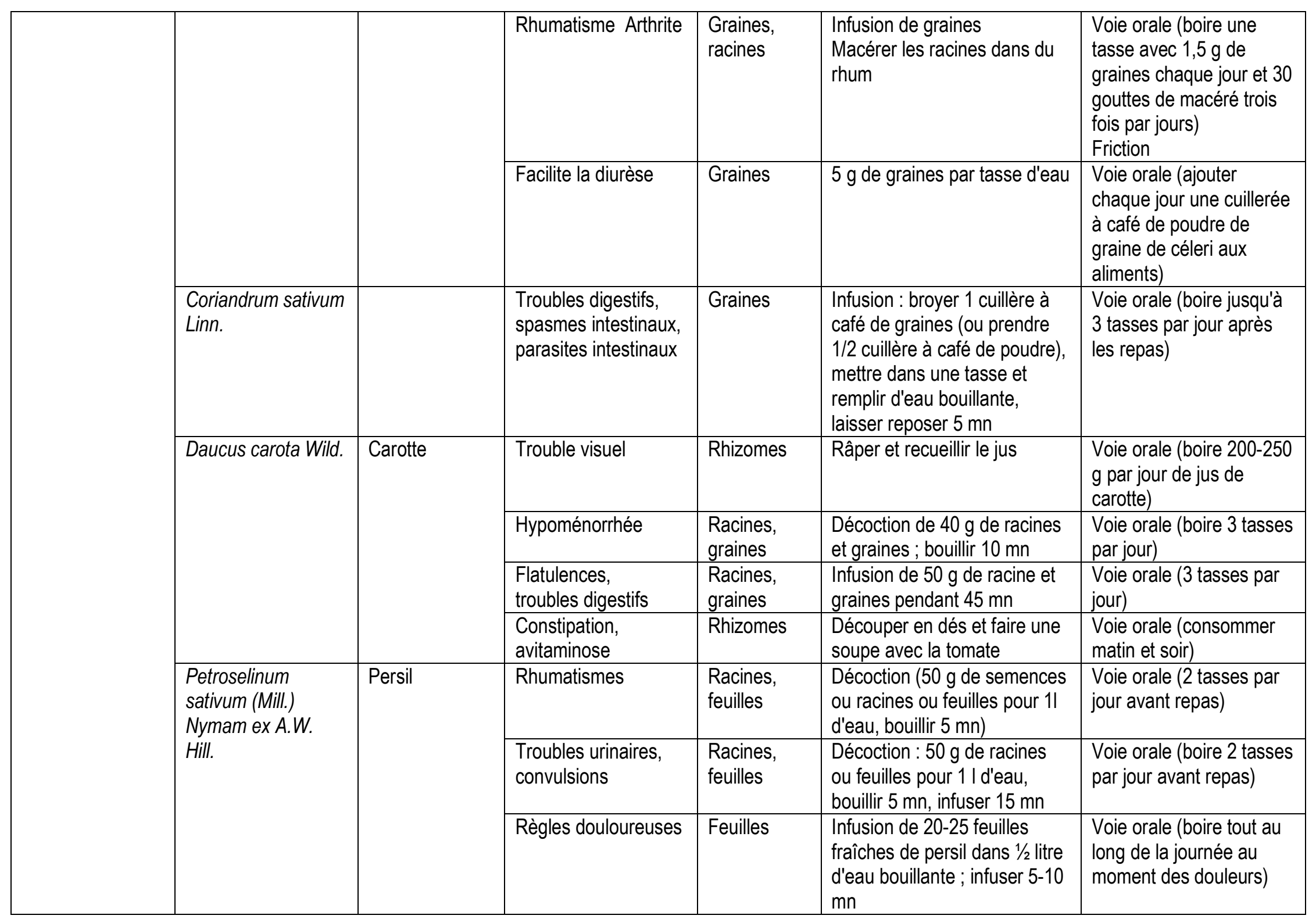




\begin{tabular}{|c|c|c|c|c|c|c|}
\hline & & & Flatulences & $\begin{array}{l}\text { Feuilles } \\
\text { fraiches, } \\
\text { graines }\end{array}$ & $\begin{array}{l}\text { Décoction (1,5 I d'eau avec } \\
750 \mathrm{ml} \text { pendant } 30 \mathrm{mn} \text {, y } \\
\text { ajouter du jus de carotte et de } \\
\text { céleri) }\end{array}$ & $\begin{array}{l}\text { Voie orale (boire } 1 \text { verre } \\
\text { tous les matins) }\end{array}$ \\
\hline & & & Mal de reins & Racines & $\begin{array}{l}\text { Décoction de } 50 \mathrm{~g} \text { de racine; } \\
\text { bouillir } 10 \mathrm{mn}\end{array}$ & $\begin{array}{l}\text { Voie orale (boire } 3 \text { tasses } \\
\text { par jour) }\end{array}$ \\
\hline & & & $\begin{array}{l}\text { Hypertension } \\
\text { artérielle }\end{array}$ & Graines & $\begin{array}{l}\text { Infusion de } 20 \mathrm{~g} \text { de graines } \\
\text { pendant } 15 \mathrm{mn} \text {; en additif } \\
\text { alimentaire } ; 2 \mathrm{~g} \text { de poudre de } \\
\text { feuilles par jour }\end{array}$ & $\begin{array}{l}\text { Voie orale (boire } 4 \text { tasses } \\
\text { par jour) }\end{array}$ \\
\hline & & & Mauvaise haleine & Feuilles, tige & $\begin{array}{l}\text { Mâcher la tige feuillée fraiche } \\
\text { et crue pendant } 1 / 4 \text { d'heure ; } \\
\text { recracher puis manger une } \\
\text { pomme }\end{array}$ & Voie orale \\
\hline & & & $\begin{array}{l}\text { Adoucissant, } \\
\text { dermatoses }\end{array}$ & Feuilles, tige & $\begin{array}{l}\text { Tremper } 50 \mathrm{~g} \text { de persil dans } \\
\text { un verre d'eau pendant } 2 \\
\text { heures ou décoction d'un gros } \\
\text { bouquet de persil qui aura } \\
\text { bouilli } 15 \mathrm{mn} \text { dans } 1 / 2 \text { litre } \\
\text { d'eau }\end{array}$ & $\begin{array}{l}\text { Usage externe (lavage } \\
\text { du visage avec cette } \\
\text { décoction tiède) }\end{array}$ \\
\hline & & & Piqures d'insectes & Feuilles & $\begin{array}{l}\text { Feuilles froissées en } \\
\text { pansement antiseptique et } \\
\text { cicatrisant }\end{array}$ & $\begin{array}{l}\text { Usage externe (faire un } \\
\text { cataplasme) }\end{array}$ \\
\hline & $\begin{array}{l}\text { Pimpinella anisum } \\
\text { Linn. }\end{array}$ & Anis & $\begin{array}{l}\text { Spasmes, } \\
\text { coliques }\end{array}$ & Graines & $\begin{array}{l}\text { Infusion de la poudre de } 1 \text { à } 2 \\
\text { graines dans une potion }\end{array}$ & $\begin{array}{l}\text { Voie orale (boire après } \\
\text { chacun des principaux } \\
\text { repas à forte dose) }\end{array}$ \\
\hline & & & Antimicrobien & $\begin{array}{l}\text { Feuilles, } \\
\text { fruits, } \\
\text { racines }\end{array}$ & Décoction & Voie orale à forte dose \\
\hline Arecaceae & $\begin{array}{l}\text { Elaeis guineensis } \\
\text { Jacq. }\end{array}$ & Palmier à huile & Transit accéléré & Noix & Extraction d'huile de noix & $\begin{array}{l}\text { Voie orale (lécher une } \\
\text { cuillerée à café) }\end{array}$ \\
\hline Areliaceae & $\begin{array}{l}\text { Panax ginseng } \\
\text { A.C. Meyer }\end{array}$ & Ginseng & Stress & Racines & $\begin{array}{l}\text { Décocter } 10 \text { g racines à } 1 / 2 \\
\text { litre d'eau ; faire bouillir } 15 \\
\mathrm{mn} \text {; laisser infuser } 10 \mathrm{mn} \text {; } \\
\text { filtrer }\end{array}$ & $\begin{array}{l}\text { Voie orale (boire } 1 \text { tasse } \\
\text { le matin, } 1 \text { tasse à midi) }\end{array}$ \\
\hline & & & Tonifiant & Racines & Décoction de 100 mg de & Voie orale (ne pas \\
\hline
\end{tabular}




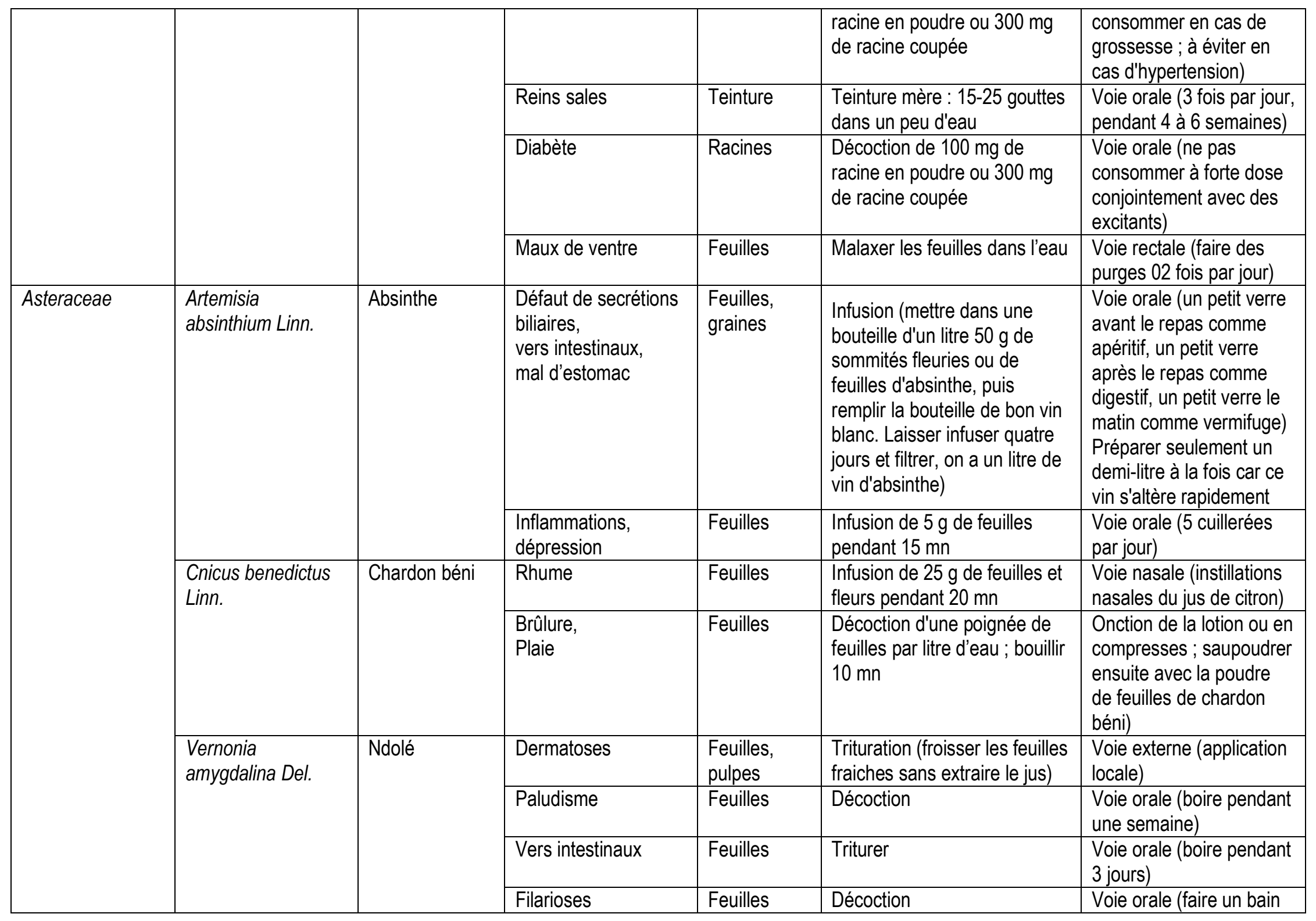


Mpondo et al.. J. Appl. Biosci. Valorisation des plantes médicinales à coumarines des marchés de Douala Est (Cameroun)

\begin{tabular}{|c|c|c|c|c|c|c|}
\hline & & & & & & et boire un verre) \\
\hline & & & Gastro-entérite & Feuilles & $\begin{array}{l}\text { Écraser et recueillir le jus, y } \\
\text { ajouter le suc extrait des } \\
\text { feuilles de goyave }\end{array}$ & $\begin{array}{l}\text { Voie orale (boire pendant } \\
5 \text { jours) }\end{array}$ \\
\hline \multirow[t]{3}{*}{ Brassicaceae } & \multirow[t]{3}{*}{$\begin{array}{l}\text { Brassica oleracea } \\
\text { (Linn.) H. Bailey }\end{array}$} & \multirow[t]{3}{*}{ Choux } & Angine & Feuilles & Expression & $\begin{array}{l}\text { Voie orale (utiliser en } \\
\text { gargarisme pendant } 2 \text { a } 3 \\
\text { jours) }\end{array}$ \\
\hline & & & Ulcère gastrique & Feuilles & Expression & $\begin{array}{l}\text { Voie orale (boire pendant } \\
\text { au moins } 3 \text { semaines) }\end{array}$ \\
\hline & & & Arthrose & Feuilles & Friction & $\begin{array}{l}\text { Appliquer un cataplasme } \\
\text { de feuille de chou avec } \\
\text { une bande de tissu sur } \\
\text { l'articulation douloureuse }\end{array}$ \\
\hline \multirow[t]{2}{*}{ Caricaceae } & \multirow[t]{2}{*}{ Carica papaya Linn. } & \multirow[t]{2}{*}{ Papayer } & Mal d'estomac & Graines & $\begin{array}{l}\text { Décoction des graines } \\
\text { écrasées dans } 1 \text { I d'eau } \\
\text { pendant } 5 \mathrm{mn}\end{array}$ & $\begin{array}{l}\text { Voie orale (Boire } 2 \text { verres } \\
\text { par jour) }\end{array}$ \\
\hline & & & Vers intestinaux & $\begin{array}{l}\text { Racines, } \\
\text { graines }\end{array}$ & $\begin{array}{l}\text { Décoction des graines } \\
\text { écrasées dans } 1 \text { I d'eau } \\
\text { pendant } 5 \mathrm{mn} \text {, y ajouter du jus } \\
\text { de citron }\end{array}$ & $\begin{array}{l}\text { Voie orale (Boire } 3 \text { verres } \\
\text { par jour après les repas) }\end{array}$ \\
\hline \multirow[t]{5}{*}{ Combretaceae } & \multirow[t]{5}{*}{$\begin{array}{l}\text { Combretum } \\
\text { micrantum G. Don }\end{array}$} & \multirow[t]{5}{*}{ Kinkeliba } & Gastro-entérite & Feuilles & Décoction & $\begin{array}{l}\text { Voie orale (boire une } \\
\text { tasse par jour) }\end{array}$ \\
\hline & & & Hypertension & Feuilles & $\begin{array}{l}\text { Décoction (faire bouillir } 20 \mathrm{~g} \\
\text { de feuilles fraîches dans un } \\
\text { litre d'eau pendant } 30 \mathrm{mn} \text { ) }\end{array}$ & $\begin{array}{l}\text { Voie orale } \\
\text { (boire toute la journée) }\end{array}$ \\
\hline & & & Hépatites & Feuilles & $\begin{array}{l}\text { Décoction (faire bouillir dans } \\
\text { l'eau des feuilles associées } \\
\text { aux racines du badamier et du } \\
\text { papayer et aux tiges feuillées } \\
\text { du goyavier) }\end{array}$ & $\begin{array}{l}\text { Voie orale (Boire } 1 \text { verre } \\
\text { matin et soir) }\end{array}$ \\
\hline & & & Plaie & Feuilles & $\begin{array}{l}\text { Les feuilles pilées appliquées } \\
\text { sur des plaies purulentes } \\
\text { activent leur cicatrisation }\end{array}$ & $\begin{array}{l}\text { Voie externe (refaire le } \\
\text { pansement une fois par } \\
\text { jour) }\end{array}$ \\
\hline & & & Diurèse difficile & Feuilles & $\begin{array}{l}\text { Décoction (faire bouillir } 20 \mathrm{~g} \\
\text { de feuilles fraîches dans un } \\
\text { litre d'eau pendant } 30 \mathrm{mn} \text { ) }\end{array}$ & $\begin{array}{l}\text { Voie orale } \\
\text { (boire toute la journée) }\end{array}$ \\
\hline
\end{tabular}


Mpondo et al.. J. Appl. Biosci. Valorisation des plantes médicinales à coumarines des marchés de Douala Est (Cameroun)

\begin{tabular}{|c|c|c|c|c|c|c|}
\hline Curcubitaceae & $\begin{array}{l}\text { Cucumis sativus } \\
\text { Linn. }\end{array}$ & Concombre & $\begin{array}{l}\text { Infections } \\
\text { microbiennes, } \\
\text { cancer, } \\
\text { obésité, } \\
\text { douleurs dentaires }\end{array}$ & Feuilles & Mâcher les feuilles crues & $\begin{array}{l}\text { Voie orale (prenez une } \\
\text { tranche de ce légume et } \\
\text { poussez-la contre le toi } \\
\text { de votre bouche avec } \\
\text { votre langue pendant } 30 \\
\text { minutes) }\end{array}$ \\
\hline Euphorbiaceae & $\begin{array}{l}\text { Manihot esculenta } \\
\text { Crantz. }\end{array}$ & Manioc & $\begin{array}{l}\text { Anémie sévère, } \\
\text { galactogène }\end{array}$ & Feuilles & $\begin{array}{l}\text { Découper dans les plats } \\
\text { laver, piler et ajouter de l'eau; } \\
\text { bouillir pendant cinq minutes } \\
\text { enfin filtré }\end{array}$ & $\begin{array}{l}\text { Voie orale (consommer } 1 \\
\text { demi-verre deux fois par } \\
\text { jour, boire le jus à } \\
\text { longueur de journée) }\end{array}$ \\
\hline \multirow[t]{6}{*}{ Fabaceae } & \multirow{2}{*}{$\begin{array}{l}\text { Desmodium } \\
\text { triflorum (Linn.) DC. }\end{array}$} & & $\begin{array}{l}\text { Infections hépatiques } \\
\text { Jaunisse } \\
\text { Asthme }\end{array}$ & Feuilles & Triturer et recueillir le jus & $\begin{array}{l}\text { Voie orale (un verre trois } \\
\text { fois par jour) }\end{array}$ \\
\hline & & & Dermatoses & Feuilles & $\begin{array}{l}\text { Triturer (froisser les feuilles et } \\
\text { appliquer) }\end{array}$ & $\begin{array}{l}\text { Appliquer sur la partie } \\
\text { infectée deux fois par } \\
\text { jour }\end{array}$ \\
\hline & Glycine max Linn. & Soja & Malnutrition & Graines & $\begin{array}{l}\text { Broyer les graines (Huile } \\
\text { alimentaire farine de soja) }\end{array}$ & Voie orale \\
\hline & $\begin{array}{l}\text { Phaseolus vulgaris } \\
\text { Linn. }\end{array}$ & Haricot rouge & $\begin{array}{l}\text { Hyperglycémie, } \\
\text { rhumatismes, } \\
\text { mal de reins, } \\
\text { hypertension } \\
\text { artérielle }\end{array}$ & Cosses & $\begin{array}{l}\text { Décoction de } 60 \mathrm{~g} \text { de } \\
\text { gousses ; bouillir } 10 \mathrm{mn}\end{array}$ & $\begin{array}{l}\text { Voie orale (boire } 3 \text { tasses } \\
\text { par jour) } \\
\text { consommer les haricots } \\
\text { verts en salade }\end{array}$ \\
\hline & \multirow[t]{2}{*}{ Senna alata Linn. } & \multirow[t]{2}{*}{ Dartrier } & Dermatoses & Feuilles & $\begin{array}{l}\text { Triturer et presser le suc de } \\
\text { feuilles sur la partie à traiter. }\end{array}$ & Voie externe \\
\hline & & & Dartre & Feuilles & Triturer puis frotter & Voie externe \\
\hline \multirow[t]{3}{*}{ Lamiaceae } & \multirow[t]{3}{*}{$\begin{array}{l}\text { Ocimun basilicum } \\
\text { Linn. }\end{array}$} & \multirow[t]{3}{*}{ Basilic } & $\begin{array}{l}\text { Spasmes, } \\
\text { infections } \\
\text { microbiennes }\end{array}$ & $\begin{array}{l}\text { Feuilles, } \\
\text { Fleurs }\end{array}$ & $\begin{array}{l}\text { Infusion ( } 20 \text { à } 30 \mathrm{~g} \text { de feuilles } \\
\text { et fleurs dans un litre d'eau) }\end{array}$ & $\begin{array}{l}\text { Voie orale (une tasse } \\
\text { après chaque repas) }\end{array}$ \\
\hline & & & Dépression nerveuse & Feuilles & $\begin{array}{l}\text { Triturer (extraire le vin des } \\
\text { feuilles) }\end{array}$ & $\begin{array}{l}\text { Voie orale (prendre } 4 \\
\text { cuillerées par jour) }\end{array}$ \\
\hline & & & Typhoïde & Feuilles & $\begin{array}{l}\text { Décoction (mélanger } 10 \mathrm{~g} \text { de } \\
\text { feuilles avec } 5 \text { poivres noires } \\
\text { et } 100 \mathrm{ml} \text { d'eau. Préparer une } \\
\text { décoction et sucrer avec } \\
\text { du sucre cristallin) }\end{array}$ & $\begin{array}{l}\text { Voie orale (boire } 3 \text { fois } \\
\text { par jour pendant } 5 \text { jours ; } \\
\text { retirer tout aliment solide } \\
\text { pendant le régime) }\end{array}$ \\
\hline
\end{tabular}




\begin{tabular}{|c|c|c|c|c|c|c|}
\hline & \multirow[t]{4}{*}{$\begin{array}{l}\text { Thymus vulgaris } \\
\text { Linn. }\end{array}$} & \multirow[t]{4}{*}{ Thym } & $\begin{array}{l}\text { Toux, } \\
\text { asthme, } \\
\text { bronchite }\end{array}$ & Feuilles & $\begin{array}{l}\text { Décoction de } 10 \mathrm{~g} \text { de } \\
\text { sommités pour } 1 / 2 \text { litre d'eau } \\
\text { en infusion pendant } 20 \mathrm{mn} \text {; } \\
\text { sucrer avec du miel }\end{array}$ & $\begin{array}{l}\text { Voie orale (Boire } 2-3 \\
\text { tasses tièdes par jour, } \\
\text { loin des repas }\end{array}$ \\
\hline & & & Dermatoses & Feuilles & $\begin{array}{l}\text { Diluer les feuilles dans de } \\
\text { l'alcool à } 90^{\circ}\end{array}$ & Application locale \\
\hline & & & $\begin{array}{l}\text { Infections de la } \\
\text { sphère buccale }\end{array}$ & Feuilles & $\begin{array}{l}2 \text { gouttes d'huile essentielle } \\
\text { dans un peu d'alcool dilué }\end{array}$ & $\begin{array}{l}\text { Voie orale (gargarisme } \\
\text { ou bain de bouche } 1 \text { à } 3 \\
\text { fois par jour) }\end{array}$ \\
\hline & & & Gastro-entérite & Feuilles & $\begin{array}{l}2 \text { gouttes d'huile essentielle + } \\
2 \text { gouttes d'huile essentielle } \\
\text { de Menthe. }\end{array}$ & $\begin{array}{l}\text { Voie orale (boire } 2 \text { fois } \\
\text { par jour) }\end{array}$ \\
\hline Lauraceae & $\begin{array}{l}\text { Persea americana } \\
\text { Mill. }\end{array}$ & Avocatier & $\begin{array}{l}\text { Maux de ventre, } \\
\text { fièvre, } \\
\text { constipation, } \\
\text { jaunisse }\end{array}$ & $\begin{array}{l}\text { Écorce, } \\
\text { feuilles, fruit, } \\
\text { graine }\end{array}$ & $\begin{array}{l}\text { Décoction ( } 30 \mathrm{gr} \text { de poudre } \\
\text { de graines par litre d'eau) }\end{array}$ & $\begin{array}{l}\text { Voie orale à prendre } \\
\text { dans les } 24 \text { heures }\end{array}$ \\
\hline \multirow[t]{3}{*}{ Liliaceae } & \multirow[t]{2}{*}{ Allium cepa Linn. } & \multirow[t]{2}{*}{ Oignon } & Gastro-entérite & Fruits & Décoction & $\begin{array}{l}\text { Voie orale } \\
\text { (boire } 3 \text { à } 4 \text { verres par } \\
\text { jour pour les adultes ; } \\
\text { Demi-verre par jour pour } \\
\text { les enfants de plus de } 5 \\
\text { ans et } \\
2 \text { cuillères à soupe pour } \\
\text { les enfants de moins de } 5 \\
\text { ans) }\end{array}$ \\
\hline & & & Dysenterie amibienne & Fruits & $\begin{array}{l}\text { Oignon cru tel quel ou macéré } \\
\text { quelques heures dans huile } \\
\text { d'olive : dans salades, } \\
\text { crudités, potages ; oignon } \\
\text { haché, macéré quelques } \\
\text { heures dans eau chaude }\end{array}$ & $\begin{array}{l}\text { Voie orale (boire matin et } \\
\text { soir avec quelques } \\
\text { gouttes de citron ; oignon } \\
\text { en poudre très digeste) }\end{array}$ \\
\hline & Allium sativum Linn. & Ail & $\begin{array}{l}\text { Maladie Cardio- } \\
\text { vasculaire, } \\
\text { diabète, } \\
\text { cancer }\end{array}$ & Gousses & $\begin{array}{l}\text { Prendre } 6 \text { à } 12 \text { gousses d'ail } \\
\text { par jour ; éplucher et mâcher } \\
3 \text { gousses en même temps }\end{array}$ & $\begin{array}{l}\text { Voie orale (consommer } \\
\text { de l'ail en salade après } \\
\text { l'avoir fait tremper } \\
\text { quelques instants dans } \\
\text { de l'eau bouillante) }\end{array}$ \\
\hline
\end{tabular}




\begin{tabular}{|c|c|c|c|c|c|c|}
\hline & & & Antibiotiques & Gousses & $\begin{array}{l}\text { L'ail doit être mâché, écrasé, } \\
\text { haché ou pressé pour libérer } \\
\text { ses propriétés antibiotiques }\end{array}$ & $\begin{array}{l}\text { Voie orale ( } 2 \text { à } 4 \text { fois par } \\
\text { jour) }\end{array}$ \\
\hline & & & $\begin{array}{l}\text { Hypertension } \\
\text { artérielle }\end{array}$ & Bulbes & $\begin{array}{l}\text { Macérer 6-7 jours dans } 1 \text { litre } \\
\text { de vin blanc sec, } 100 \mathrm{~g} \text { de } \\
\text { bulbes frais pilés }\end{array}$ & $\begin{array}{l}\text { Voie orale (boire } 2 \text { verres } \\
\text { par jour loin des repas). }\end{array}$ \\
\hline & & & Toux & Gousses & $\begin{array}{l}\text { Macérer } 100 \mathrm{~g} \text { de gousses } \\
\text { fraîches écrasées dans } 1 \text { litre } \\
\text { de vin blanc sec pendant } 8 \\
\text { jours }\end{array}$ & $\begin{array}{l}\text { Voie orale (boire 3-4 } \\
\text { cuillerées par jour) }\end{array}$ \\
\hline & & & Infection sanguine & Gousses & $\begin{array}{l}\text { Macérer } 25 \mathrm{~g} \text { de gousse } \\
\text { hachée dans } 100 \mathrm{~g} \text { d'alcool à } \\
70^{\circ} \text { pendant } 15 \text { jours }\end{array}$ & $\begin{array}{l}\text { Voie orale (prendre } 20 \\
\text { gouttes par jour dans de } \\
\text { l'eau ou dans toute autre } \\
\text { boisson ou nourriture) }\end{array}$ \\
\hline & & & Démangeaison & Gousses & $\begin{array}{l}\text { Décoction de } 6 \text { gousses d'ail } \\
\text { par litre d'eau ; bouillir } 20 \mathrm{mn}\end{array}$ & $\begin{array}{l}\text { Voie externe (lavage } \\
\text { avec la décoction) }\end{array}$ \\
\hline & Aloe Vera Linn. & Aloes & $\begin{array}{l}\text { Dermatoses (gale, } \\
\text { acné, } \\
\text { eczéma) }\end{array}$ & La sève & $\begin{array}{l}\text { Laver les feuilles et frotter le } \\
\text { gel }\end{array}$ & $\begin{array}{l}\text { Voie externe (frotter la } \\
\text { sève sur la partie malade } \\
\text { après le bain jusqu'à } \\
\text { disparition complète de la } \\
\text { gale) }\end{array}$ \\
\hline & & & $\begin{array}{l}\text { Mal de reins, } \\
\text { infection sanguine }\end{array}$ & Feuille & $\begin{array}{l}\text { Décoction (découper la feuille } \\
\text { en dé ; laisser macérer dans } \\
\text { l'eau pendant une journée) }\end{array}$ & $\begin{array}{l}\text { Voie orale (boire un verre } \\
\text { matin et soir pendant une } \\
\text { semaine) }\end{array}$ \\
\hline & & & Muguet & Feuilles & Extraction du gel ou du suc & $\begin{array}{l}\text { Voie orale (1 cuillère à } \\
\text { soupe de suc frais } 2 \text { fois } \\
\text { par jour) }\end{array}$ \\
\hline & & & Cosmétique & Sève & $\begin{array}{l}\text { Mélanger la sève avec I huile } \\
\text { de jojoba }\end{array}$ & $\begin{array}{l}\text { Appliquer au visage au } \\
\text { coucher une fois par } \\
\text { semaine }\end{array}$ \\
\hline & & & Maux de ventre & Feuille & $\begin{array}{l}\text { Décoction (découper la feuille } \\
\text { puis malaxer dans de l eau) }\end{array}$ & $\begin{array}{l}\text { Voie rectale (faire } 2-3 \\
\text { poires) }\end{array}$ \\
\hline Malvaceae & $\begin{array}{l}\text { Albelmoschus } \\
\text { esculentus (Linn.) } \\
\text { Moench }\end{array}$ & Gombo & Constipation & $\begin{array}{l}\text { Fleurs, } \\
\text { fruits, } \\
\text { feuilles }\end{array}$ & Décoction & $\begin{array}{l}\text { Voie rectale ( } 1 \text { purge par } \\
\text { jour pendant } 3 \text { jours) }\end{array}$ \\
\hline & & & Antispasmodique & Fruits & Décoction & Voie orale ( 2 cuillerées à \\
\hline
\end{tabular}


Mpondo et al.. J. Appl. Biosci. Valorisation des plantes médicinales à coumarines des marchés de Douala Est (Cameroun)

\begin{tabular}{|c|c|c|c|c|c|c|}
\hline & & & & & & $\begin{array}{l}\text { café délayées dans du } \\
\text { potage chaud) }\end{array}$ \\
\hline \multirow[t]{3}{*}{ Myrtaceae } & \multirow[t]{3}{*}{$\begin{array}{l}\text { Eucalyptus sailgna } \\
\text { Smith }\end{array}$} & \multirow[t]{3}{*}{ Eucalyptus } & Lourdeur des jambes & Feuilles & $\begin{array}{l}\text { Infusion de } 30 \mathrm{~g} \text { de feuilles } \\
\text { adultes pendant } 10 \mathrm{mn}\end{array}$ & $\begin{array}{l}\text { Voie orale (boire } 3 \text { tasses } \\
\text { par jour avec du miel) }\end{array}$ \\
\hline & & & $\begin{array}{l}\text { Dysenterie } \\
\text { amibienne, } \\
\text { paludisme }\end{array}$ & Feuilles & $\begin{array}{l}\text { Macérer les feuilles fraiches } \\
\text { dans un litre d'eau }\end{array}$ & $\begin{array}{l}\text { Voie orale (boire un verre } \\
\text { matin midi et soir) }\end{array}$ \\
\hline & & & Plaies chroniques & Feuilles & $\begin{array}{l}\text { Ajouter des feuilles des } \\
\text { rameaux âgés à } 1 \text { grand bol } \\
\text { d'eau bouillante }\end{array}$ & $\begin{array}{l}\text { Fumigation (respirer les } \\
\text { vapeurs pendant } \\
\text { quelques minutes) }\end{array}$ \\
\hline \multirow[t]{4}{*}{ Pedaliaceae } & \multirow[t]{4}{*}{$\begin{array}{l}\text { Sesamum indicum } \\
\text { Linn. }\end{array}$} & \multirow[t]{4}{*}{ Sésame } & Hypercholestérolémie & Graines & $\begin{array}{l}\text { Tremper les graines dans } \\
\text { l'eau pendant } 15 \mathrm{mn} \text {, les } \\
\text { égoutter et les griller }\end{array}$ & $\begin{array}{l}\text { Voie orale (boire } 02 \text { ou } \\
03 \text { cuillerées à café après } \\
\text { le petit déjeuner et le } \\
\text { déjeuner) }\end{array}$ \\
\hline & & & $\begin{array}{l}\text { Hypertension } \\
\text { artérielle }\end{array}$ & Graines & Bouillir 9 à $15 \mathrm{~g}$ & $\begin{array}{l}\text { Voie orale (boire deux } \\
\text { verres par jour) }\end{array}$ \\
\hline & & & $\begin{array}{l}\text { Maladies } \\
\text { cardiovasculaires }\end{array}$ & Graines & Décoction & $\begin{array}{l}\text { Voie orale (consommer } \\
\text { une cuillère à soupe de } \\
\text { graines trois fois par jour } \\
\text { pendant deux à quatre } \\
\text { semaines) }\end{array}$ \\
\hline & & & Déficience hépatique & Graine & Concasser & Voie orale \\
\hline \multirow[t]{4}{*}{ Poaceae } & \multirow[t]{3}{*}{ Zea mays Linn. } & \multirow[t]{3}{*}{ Maïs } & Cystite & $\begin{array}{l}\text { Barbe du } \\
\text { mais }\end{array}$ & Infusion & $\begin{array}{l}\text { Voie orale (boire } 500 \mathrm{ml} \\
\text { par jour) }\end{array}$ \\
\hline & & & $\begin{array}{l}\text { Traitement adjuvant } \\
\text { du lymphoedème }\end{array}$ & Graines & Infusion des stigmates & $\begin{array}{l}\text { Voie orale (03 à } 05 \\
\text { tasses chaudes ou } \\
\text { froides par jour) }\end{array}$ \\
\hline & & & $\begin{array}{l}\text { Diurétique, } \\
\text { troubles biliaires, } \\
\text { troubles urinaires }\end{array}$ & $\begin{array}{l}\text { Graines, } \\
\text { barbes }\end{array}$ & $\begin{array}{l}\text { Infusion ou décoction des } \\
\text { graines dans l'eau }\end{array}$ & $\begin{array}{l}\text { Voie orale (boire } 500 \mathrm{ml} \\
\text { (barbe de maïs) ; gélules } \\
\text { d'extrait sec) }\end{array}$ \\
\hline & Oryza sativa Linn. & Riz & $\begin{array}{l}\text { Diabète, } \\
\text { gastro-entérite }\end{array}$ & Graines & $\begin{array}{l}\text { Faire bouillir le riz très } \\
\text { lentement dans } 1 \text { I d'eau }\end{array}$ & $\begin{array}{l}\text { Voie orale (consommer le } \\
\text { riz et boire ou utiliser } \\
\text { l'eau de cuisson pour } \\
\text { lavements) }\end{array}$ \\
\hline Rubiaceae & $\begin{array}{l}\text { Galium odoratum } \\
\text { (Linn.) Scop }\end{array}$ & $\begin{array}{l}\text { Belle dame de } \\
\text { nuit }\end{array}$ & $\begin{array}{l}\text { Sédatif, } \\
\text { diurétique, }\end{array}$ & Fleurs & $\begin{array}{l}\text { Infuser } 1 \text { cuillerée à dessert } \\
\text { de fleurs bien séchée par }\end{array}$ & $\begin{array}{l}\text { Voie orale (boire } 3 \text { tasses } \\
\text { par jour) }\end{array}$ \\
\hline
\end{tabular}




\begin{tabular}{|c|c|c|c|c|c|c|}
\hline & & & spasmodique & & $\begin{array}{l}\text { tasse d'eau ; infuser } 3 \text { à } 5 \mathrm{mn} \\
\text { (ne pas faire bouillir) }\end{array}$ & \\
\hline & & & Antiseptique & $\begin{array}{l}\text { Feuilles, } \\
\text { fleurs, tige }\end{array}$ & $\begin{array}{l}\text { Infusion de } 50 \mathrm{~g} \text { de plante } \\
\text { entière pendant } 8 \text { - } 10 \text { heures ; } \\
\text { par cuillerées ; ou plante bien } \\
\text { séchée : infusion : } 1 \text { cuillerée } \\
\text { à dessert de fleurs par tasse } \\
\text { d'eau ; infuser } 3 \text { à } 5 \mathrm{mn} \text { (ne } \\
\text { pas faire bouillir): }\end{array}$ & $\begin{array}{l}\text { Voie orale (boire } 3 \text { tasses } \\
\text { par jour) }\end{array}$ \\
\hline \multirow[t]{7}{*}{ Rutaceae } & $\begin{array}{l}\text { Citrus aurantifolia } \\
\text { (Christm.) Swingle }\end{array}$ & Oranger & Convulsions & $\begin{array}{l}\text { Feuilles } \\
\text { fleurs }\end{array}$ & $\begin{array}{l}\text { Faire une décoction ou un } \\
\text { sirop à base des feuilles et de } \\
\text { fleurs }\end{array}$ & $\begin{array}{l}\text { Voie orale (boire une } \\
\text { tasse matin et soir) }\end{array}$ \\
\hline & \multirow[t]{4}{*}{$\begin{array}{l}\text { Citrus grandis } \\
\text { Osbeck }\end{array}$} & \multirow[t]{4}{*}{ Pamplemousse } & Hémorragie & Écorces & $\begin{array}{l}\text { Macération (Réduire } 5 \mathrm{~g} \\
\text { d'écorce en fins fragments } \\
\text { introduire un litre d'eau froide. } \\
\text { Porter à ébullition pendant } 15 \\
\text { mn, filtrer) }\end{array}$ & $\begin{array}{l}\text { Voie orale (boire1-3 } \\
\text { tasses par jours) }\end{array}$ \\
\hline & & & $\begin{array}{l}\text { Convulsion des } \\
\text { enfants }\end{array}$ & Feuilles & Poudre de feuille & Inhaler par voie nasale \\
\hline & & & Fatigue & Fruits & Faire un jus à base des fruits & $\begin{array}{l}\text { Voie orale (boire un verre } \\
\text { de } \\
\text { jus de pamplemousse } \\
\text { avant chaque repas) }\end{array}$ \\
\hline & & & Troubles digestifs & Fruits & Faire un jus à base des fruits & $\begin{array}{l}\text { Voie orale (boire } 3 \text { verres } \\
\text { par jour avant les repas) }\end{array}$ \\
\hline & \multirow[t]{2}{*}{ Citrus limon Linn. } & \multirow[t]{2}{*}{ Citron } & $\begin{array}{l}\text { Infections } \\
\text { microbiennes, } \\
\text { troubles digestifs, } \\
\text { perte d'appétit, } \\
\text { intoxication, } \\
\text { gastro-entérite }\end{array}$ & $\begin{array}{l}\text { Fruits, } \\
\text { pépins }\end{array}$ & $\begin{array}{l}\text { Pépins broyés et macéré } \\
\text { avec du miel ; jus d'un demi } \\
\text { citron dans un demi verre } \\
\text { d'eau ou décoction de } 3 \text { citron } \\
\text { avec de la citronnelle }\end{array}$ & $\begin{array}{l}\text { Voie orale (boire } 1 \text { verre } \\
\text { matin et soir) }\end{array}$ \\
\hline & & & $\begin{array}{l}\text { Scorbut, } \\
\text { athérosclérose, } \\
\text { obésité }\end{array}$ & Fruits & $\begin{array}{l}100-200 \mathrm{~g} \text { de jus de citron } \\
\text { pendant et en dehors des } \\
\text { repas, par petites doses dans } \\
1 \text { peu d'eau }\end{array}$ & $\begin{array}{l}\text { Voie orale (boire le jus de } \\
2-3 \text { citrons pendant } 12 \\
\text { jours consécutifs) }\end{array}$ \\
\hline
\end{tabular}




\begin{tabular}{|c|c|c|c|c|c|c|}
\hline & & & $\begin{array}{l}\text { Rhumatismes, } \\
\text { fièvre, } \\
\text { fatigue, } \\
\text { convulsions }\end{array}$ & Fruits & $\begin{array}{l}1 / 2 \text { citron par jour jusqu'à } 8-10 \\
\text { citrons; ne pas sucrer avec } \\
\text { du sucre mais avec du miel, } \\
\text { verser le jus de } 6 \text { citrons dans } \\
1 \text { récipient d'eau chaude, y } \\
\text { tremper les pieds } 10-15 \mathrm{mn} \text {; } \\
\text { en même temps, consommer } \\
\text { quelques fines lamelles d'ail }\end{array}$ & $\begin{array}{l}\text { Voie orale (boire le jus } \\
\text { dilué dans } 50 \% \text { d'eau ; } \\
\text { diminuer les doses } \\
\text { progressivement) }\end{array}$ \\
\hline & & & $\begin{array}{l}\text { Irritation de la gorge, } \\
\text { angine, toux }\end{array}$ & Fruits & Extraire le jus de citron & $\begin{array}{l}\text { Voie orale (boire une } \\
\text { tasse de jus relevé de } \\
\text { miel ; en gargarismes) }\end{array}$ \\
\hline & & & Panaris & Fruits & Chauffer le fruit à feux doux & $\begin{array}{l}\text { Mettre le fruit cuit sur la } \\
\text { partie atteinte }\end{array}$ \\
\hline & & & Filaire & Fruits & $\begin{array}{l}\text { Frotter la partie atteinte avec } \\
\text { du jus de citron }\end{array}$ & Voie externe \\
\hline & & & Sinusite & Fruits & $\begin{array}{l}\text { Faire bouillir } 1 \text { bol d'eau ; y } \\
\text { ajouter le jus de } 2 \text { citrons, du } \\
\text { poivre et } 1 \text { pincée de gros } \\
\text { sel ; retirer }\end{array}$ & $\begin{array}{l}\text { Inhaler la vapeur sous } \\
\text { une serviette }\end{array}$ \\
\hline & Citrus medica Linn. & Citron & Angine & Fruits & Décoction & $\begin{array}{l}\text { Voie orale (faire un } \\
\text { gargarisme avec le suc) }\end{array}$ \\
\hline & & & $\begin{array}{l}\text { Vers intestinaux, } \\
\text { gastro-entérite }\end{array}$ & Graines & Décoction des graines pilées & Voie orale \\
\hline & & & $\begin{array}{l}\text { Convulsions, } \\
\text { mal de nerfs, } \\
\text { épilepsie }\end{array}$ & Fruit & $\begin{array}{l}300-400 \mathrm{~g} \text { par jour de jus du } \\
\text { fruit dilué dans } 50 \% \text { d'eau }\end{array}$ & Voie orale \\
\hline Urticacée & Urtica dioica Linn. & & Hémorragie & $\begin{array}{l}\text { Plantes } \\
\text { entière }\end{array}$ & $\begin{array}{l}\text { Décoction de } 80 \mathrm{~g} \text { de plante } \\
\text { entière ; bouillir } 10 \mathrm{mn}\end{array}$ & Voie orale \\
\hline & & & Anémie & Feuilles & $\begin{array}{l}\text { Décoction de } 50 \mathrm{~g} \text { de feuilles; } \\
\text { bouillir } 12 \mathrm{mn}\end{array}$ & $\begin{array}{l}\text { Voie orale (boire 2-3 } \\
\text { tasses par jour) }\end{array}$ \\
\hline & & & $\begin{array}{l}\text { Rhumatismes, } \\
\text { gastro-entérite } \\
\text { Mal de reins }\end{array}$ & $\begin{array}{l}\text { Plante } \\
\text { entière }\end{array}$ & $\begin{array}{l}\text { Infusion (verser une tasse } \\
\text { d'eau bouillante sur une } \\
\text { cuillerée de la plante } \\
\text { desséchée et émiettée ; } \\
\text { laisser reposer } 20 \mathrm{mn}\end{array}$ & $\begin{array}{l}\text { Voie orale (prendre } 4 \text { ou } \\
5 \text { tasses par jour. Cette } \\
\text { cure dure généralement } \\
20 \text { jours par mois, } \\
\text { recommencé le mois } \\
\text { suivant, si nécessaire) }\end{array}$ \\
\hline
\end{tabular}




\begin{tabular}{|l|l|l|l|l|l|l|}
\hline & & $\begin{array}{l}\text { hypertension } \\
\text { artérielle } \\
\text { Diabète }\end{array}$ & Feuilles & $\begin{array}{l}\text { Décoction de 100 g de feuilles } \\
\text { dans de l'eau }\end{array}$ & $\begin{array}{l}\text { Voie orale (boire 3 fois } \\
\text { par jour) }\end{array}$ \\
\hline Zingiberaceae & $\begin{array}{l}\text { Afromomum } \\
\text { excarpum (Sims) } \\
\text { Hepper }\end{array}$ & Mbongo & Hernie ombilical & Graines & $\begin{array}{l}\text { Écraser les graines, les } \\
\text { mélanger avec des feuilles de } \\
\text { Ocimum gratissimum et le roi } \\
\text { des herbes }\end{array}$ & $\begin{array}{l}\text { Voie orale (boire le } \\
\text { décocté ou manger pas } \\
\text { moins de 9 graines) }\end{array}$ \\
\hline
\end{tabular}


Parmi les 38 espèces végétales à coumarines recensées dans les marchés Est de la ville de Douala, 5 sont locales et 33 sont exotiques. Ces espèces comptent 34 genres repartis dans 21 familles dont la plus représentée est celle des Apiaceae. Elle est suivie de la famille des Fabaceae puis celle des Rutaceae. Bourgaud et al. (1989) trouvent que ces familles sont avec celle des Moraceae les familles dans lesquelles la plupart des plantes à coumarines ont été décrites. Les espèces locales sont: Aframomum escarpum, Allium cepa, Elaeis guineensis, Ocimum basilicum et Vernonia amygdalina. Les espèces exotiques sont: Albelmoschus esculentus, Allium sativum, Aloe Vera, Anethum graveolens, Apium graveolens, Artemisia absinthium, Brassica oleracea, Carica papaya, Citrus aurantifolia, Citrus grandis, Citrus limon, Citrus medica, Cnicus benedictus, Combretum micrantum, Coriandrum sativum, Cucumis sativus, Daucus carota, Desmodium triflorum, Eucalyptus sailgna, Galium odoratum, Glycine max, Manihot esculenta, Oryza sativa, Panax ginseng, Persea americana, Petroselinum sativum, Phaseolus vulgaris, Pimpinella anisum, Senna alata, Sesamum indicum, Thymus vulgaris, Urtica dioica et Zea mays. Les vendeuses se ravitaillent dans les champs, les forêts secondarisées, les jardins de cases, les vergers et les jachères. Ces espèces sont pour la plupart cultivées et les feuilles, fruits et graines sont généralement utilisées en cuisine comme condiments ou comme épices (Filliat, 2012). Ces organes sont également les plus sollicités dans les préparations médicamenteuses. Selon Brown (1970), les organes reproducteurs et les parties exposées à la prédation constituent les organes de stockage par excellence des coumarines. La décoction est le mode de préparation le plus sollicité $(38,13 \%)$. Ce résultat se rapproche de celui établi par N'Guessan et al., (2009) qui indiquent que la décoction est le mode de préparation le plus sollicité dans $42,30 \%$ des cas. L'ingestion orale $(74,54 \%)$ est la voie d'administration par excellence des médicaments à base de plantes à coumarines. Les coumarines sont des phytoalexines aux fonctions écologiques nombreuses, en rapport avec leur large spectre de modes d'action biologique. Elles confèrent aux plantes qui en synthétisent un certain avantage adaptatif (Gravot, 2002). Ce sont des inhibiteurs de germination et de croissance vis-à-vis des plantes concurrentes. Elles sont également des molécules toxiques et phototoxiques dont la présence préserve dans une certaine mesure des prédateurs et de microorganismes pathogènes. Par ailleurs, les coumarines sont des synergistes vis-à-vis d'autres phytoalexines toxiques (Gravot, 2002). Parmi les maladies soignées par les plantes à coumarines recensées dans les marchés, les maladies de l'appareil digestif sont les plus importantes $(33,33 \%)$. En effet, les plantes à coumarines et plus spécifiquement celles de la famille des Apiaceae ont la capacité de favoriser l'expulsion des gaz intestinaux entraînant une diminution des ballonnements et des flatulences. Anethum graveolens, Apium graveolens, Daucus carota et Petroselinum sativum stimulent la sécrétion des glandes digestives entraînant une bonne dégradation des aliments et limitent les fermentations indésirables. Elles augmentent la production stomacale d'acide contribuant à une bonne désinfection du bol alimentaire (Filliat, 2012). Coriandrum sativum, Pimpinella anisum et Ocimum basilicum ont une importante activité antispasmodique et des propriétés antimicrobiennes marquées (Bruneton, 2009). Les coumarines de ces plantes ont un effet spasmolytique réduisant les spasmes et le météorisme abdominal. Par ailleurs, ces coumarines sont à même de réduire la multiplication des bactéries pathogènes et ainsi, empêcher la formation de gaz et de métabolites toxiques au niveau intestinal (Harkati, 2011). En effet, le caractère lipophile des coumarines leur permet comme tous autres phénols de se lier aux membranes cellulaires des microorganismes et d'inhiber les échanges d'électrons membranaires lors de la phosphorylation oxydative freinant le métabolisme cellulaire (Teuscher et al., 2005 ; Kaloustian et al., 2008). Les coumarines agissent comme les flavonoïdes, elles ont la capacité de prévenir la peroxydation des lipides membranaires et de capter les radicaux hydroxyles, superoxydes et peroxyles (Caesar, 2007 ; Mpondo et al., 2012). Allium sativum et Sesamum indicum sont sollicitées pour traiter les scléroses, l'hypertension artérielle, les maladies cardiovasculaires. Les coumarines comme les autres phénols antioxydants agissent comme des poubelles à radicaux libres en prévenant et en arrangeant les dommages causées par les ROS (Reactive Oxygen Species-Espèces Réactives Oxygénées) (Fraga et al., 1996). Les dérivés coumariniques de nombreuses plantes possèdent des activités anticoagulantes, qui entraînent des hémorragies chez les herbivores qui en consomment (Caesar, 2007). Le 4-hydroxy-3-[1-(4-nitrophényl)-3oxobutyl] coumarine, appelé usuellement 
acénocoumarol, est antagoniste de la vitamine $\mathrm{K}$ et inhibiteur de la synthèse des facteurs de la coagulation vitamino-K-dépendants (Bruneton, 2009). Le dicoumarol et l'esculoside, tous deux veinotoniques et vasculoprotecteurs (Hostettmann, 1997) sont également responsables de cette activité et sont utilisées dans la thérapie des maladies thromboemboliques. Les coumarines ont des propriétés antipyrétique, analgésique, sédative, antioedèmateuse et anti convulsivante

\section{CONCLUSION}

II ressort de cette étude dont l'objectif était de recenser les plantes à coumarines et leurs différents usages que, ces plantes dont l'odeur agréable rappelle la vanilline sont pour la plupart des plantes cultivées dont les feuilles, fruits et graines sont généralement utilisés en cuisine comme condiments ou comme épices. Elles appartiennent à plusieurs familles dont les plus importantes sont celles des Apiaceae, des Fabaceae et des Rutaceae. Les coumarines ont des fonctions écologiques nombreuses et confèrent aux plantes qui en synthétisent un certain avantage adaptatif. Les maladies de l'appareil digestif sont les plus importantes parmi les maladies soignées par les plantes à coumarines recensées et la voie orale est la forme

\section{REFERENCES BIBLIOGRAPHIQUES}

Adjanohoun E, Cusset G, Issa LO, Keita A, Lebras M, Lejoly J, Waechter P. 1989. Banque de données de médecine traditionnelle et de pharmacopée (pharmel). Notice pour la récolte et l'entrée des données, ACCT, Paris.

Adjanohoun E, Cusset G, Issa LO, Keita A, Lebras M, Lejoly J. 1994. Banque de données de médecine traditionnelle et de pharmacopée (Pharmel). Notice pour la collecte et l'entrée des données, seconde édition. A.C.C.T., Paris.

Adjanohoun E. 2000. La biodiversité face au développement des industries pharmaceutiques africaines. In : Réseau des " espèces ligneuses médicinales ", Eyog Matig O, Adjanohoun E, de Souza S et Sinsin B (eds). Compte rendu de la première réunion du réseau tenue 15-17 décembre 1999 à la station IITA Cotonou, Bénin, pp88-103.

Adomou AC, Yedomonhan H, Djossa B, Legba SI, Oumorou M, Akoegninou A. 2012. Étude Ethnobotanique des plantes médicinales
(Gupta et al., 1980). Elles sont indiquées dans les cas de lymphoedème du membre supérieur après traitement radiochirurgical du cancer du sein (Harkati, 2011). Les coumarines présentes dans les espèces du genre Citrus (auraptène, subérosine) (Bruneton, 2009) semblent avoir une forte activité anti convulsivante de même que ceux de la famille des Apiaceae. Elles semblent également être responsables de l'activité duirétique.

pharmaceutique la plus sollicitée. Les coumarines ont la capacité de prévenir la peroxydation des lipides membranaires et de capter les radicaux hydroxyles, superoxydes et peroxyles. Elles ont des propriétés antipyrétique, analgésique, sédative, antioedèmateuse et anti convulsivante. Les plantes à coumarines ont la capacité de favoriser l'expulsion des gaz intestinaux entraînant une diminution des ballonnements et des flatulences. Elles ont un effet spasmolytique. La connaissance de toutes ces propriétés devrait davantage orienter les populations de la ville de Douala à intégrer ces plantes dans leur alimentation afin de renforcer la défense immunitaire et réduire les risques de maladies dégénératives.

vendues dans le marché d'Abomey-Calavi au Bénin. Int. J. Biol. Chem. Sci. 6: 745772.

Béllé BJ. 1992. Lexique des essences des forêts denses centrafricaines. Ministère des eaux, forêts, Pêche et du Tourisme.

Biyiti LF, Meko'o DJL, Tamzc V, Amvam Zollo PH. 2004. Recherche de l'activité antibactérienne de quatre plantes médicinales camerounaises. Pharm. Med. Trad. Afr. 13: 1120.

Bourgaud F, Allard N, Guckert A, Forlot P. 1989. Natural sources of furocoumarins (psoralens). In Psoralens : Past, Present and Future of Photochemoprotection and other biological activities, T.B. Fitzpatrick, Forlot, P., Pathak, M.A., Urbach, F., ed (Paris : John Libbey Eurotext), pp. 219230.

Brown SA. 1970. Biosynthesis of furanocoumarins in parnsips. Phytochemistry 9: 2471-2475.

Bruneton J. 2009. Pharmacognosie, phytochimie, plantes médicinales, 4e édition, Tec \& Doc, Paris, 1269 pp. 
Caesar LN. 2007. Étude de la phytochimie et des activités biologiques de deux plantes utilisées en médecine traditionnelle gabonaise : Terminalia catappa Linn. (Combretaceae) et Kalanchoe crenata (Andr.) Haw. (Crassulaceae). Thèse de Doctorat Université de Bamako Mali 142p.

Cunningham AB. 1993. African medicinal plants. Setting priorities at the interface between conservation and primary healthcare. People and Plants Working Paper, (vol 1). UNESCO : Paris ; 50.

Dibong SD, Mpondo Mpondo E, Ngoye A, Kwin NF. 2011. Plantes médicinales utilisées par les populations bassa de la région de Douala au Cameroun. International Journal of Biological and Chemical Sciences 5: 1105-1117.

Din N, Senger P, Priso JR, Dibong SD, Amougou A. 2008. Logging activities in mangrove forests. A case study of Douala Cameroon. Africa journal in environmental science and technology 2: 22-30.

Filliat P. 2012. Les plantes de la famille des Apiacées dans les troubles digestifs. Thèse de Doctorat Université de Grenoble France 129p.

Fraga CG, Motchnik PA, Shigenaga MK, Helbock HJ, Jacob RA, Ames BN. 1996. Ascorbic acid proteids against endogenous oxidative DNA damage in human sperm. Proc. Nat. Acad. Sci. 88: 11003-11006.

Gravot A. 2002. Étude de P450s impliqués dans la biosynthèse des furocoumarines chez Ruta graveolens. Thèse de Doctorat Université de NANCY-COLMAR France 210p.

Gray Al et Waterman PG. 1978 : Phytochemistry 17: 845 .

Gupta MB, Nath R. 1980. Anti inflammatory and antipyretic activities of sitosterol, Planta medica 39: 157-163.

Hamburger M. et Hostettmann K. 1991.Bioactivity in plants : the link between phytochemistry and medicine. Phytochem. 30: 3864-3874.

Harkati B. 2011. Valorisation et identification structurale des principes actifs de la plante de la famille Asteraceae: Scorzonera undulata. Thèse de Doctorat, Université de Mentouri-constantine Algerie. 129p.

Hostettmann K. 1997. Tout Savoir sur le Pouvoir des Plantes Sources de Médicaments. Editions Favre, Lausanne 93:104-135.
Jiofack T, Fokunang C, Guedje N, Kemeuze V, Fongnzossie E, Nkongmeneck BA, Mapongmetsem PM, Tsabang N. 2010. Ethnobotanical uses of medicinals plants of two ethnoecological regions of Cameroon. International Journal of Medicine and Medical Sciences 2: 60-79.

Kaloustian J, Chevalier J, Mikail C, Martino M, Abou L, Vergnes MF. 2008. Étude de six huiles essentielles : composition chimique et activité antibactérienne, Phytothérapie $6: 160-4$.

Koné D. 2009. Enquête ethnobotanique de six plantes médicinales maliennes extraction, identification d'alcaloïdes caractérisation, quantification de polyphénols : étude de leur activité antioxydante. Thèse de Doctorat Université de Bamako, Mali. 157p.

Letouzey R. 1970. Manuel de botanique forestière, Afrique Tropicale. Tome 2, CTFT, Sainte Marie. France.

Letouzey R. 1985. Carte phytogéographique du Cameroun au 1/500000 no4. IRA-ICIV, Université de Toulouse, France. 240p.

Mpondo Mpondo E, Dibong SD, Ladoh YCF, Priso RJ, Ngoye A. 2012. Les plantes à phénols utilisées par les populations de la ville de Douala. Journal of Animal \&Plant Sciences 15: 2083-2098.

N'Guessan K, Kadja B, Zirihi GN, Traoré D, Akéassi L. 2009. Screening phytochimique de quelques plantes médicinales ivoiriennes. Sciences \& Nature 6: 1-15.

Olsen CS. 2005. Trade and conservation of Himalayan medicinal plants: Nardostachys grandiflora DC and Neopicrorhiza scrophulariiflora (Pennell) Hong. Biol. Conserv., 125: 505-514.

OMS. 2000. Stratégie de l'OMS pour la médecine traditionnelle pour 2002-2005, Genève. 78 p.

Pei S. 2001. Ethnobotanical Approaches of traditional medicine studies: some experiences from Asia. Pharm. Bot. 39: 74-79.

Priso RJ, Nnanga JF, Etame J, Din N, Amougou A. 2011. Les produits forestiers non ligneux d'origine végétale : valeur importance dans quelques marchés de la région du littoral Cameroun. J. of Appl. Biosci. 40 : 2715-2726.

Teuscher E, Anton R., Lobstein A. 2005. Plantes aromatiques : épices, aromates, 
Mpondo et al.. J. Appl. Biosci. Valorisation des plantes médicinales à coumarines des marchés de Douala Est (Cameroun)

condiments et huiles essentielles, Tec \& Doc, Paris. $522 \mathrm{p}$.

Weterman PG, Grundon MF. 1983. Chemistry, Chemical Taxonomy of The Rutales, Academic Press London-New York.
WHO. 2010. International Statistical Classification of Diseases and Related Health Problems. 10th Revision. Instruction manual vol 2. Edition. WHO, Geneva. $199 p$. 